\title{
Influence of Inoculation Site of Combined Oil-Adjuvanted Vaccine on the Antibody Response in Chickens
}

\author{
Kazuhiro DEGUCHI, Takashi HONDA, Kazuo MATSUO, Hideo FUJIKAWA, Toshinori IWAMOTO ${ }^{1)}$ and \\ Yoshihiro SAKANOUE2) \\ The Chemo-Sero-Therapeutic Research Institute, 1-6-1 Okubo, Kumamoto 860-8568 and ${ }^{1}$ Kagoshima-Pref. Agricultural Cooperative \\ Federation 15 Kamoike-shinmachi, Kagoshima 890-0064, and ${ }^{21}$ Kagoshima-Kumiai-Chicken Foods Co.,Ltd., 15 Kamoike-shinmachi, \\ Kagoshima 890-0064, Japan
}

(Received 9 December 1997/Accepted 20 March 1998)

ABSTRACT. Inactivated oil-adjuvanted vaccines for ND, IB, and IC serotypes A and C (OILVAX NB ${ }_{2}$ AC) have been marketed from 1993 .
In the outdoors, various inoculation sites have been used in chickens because to make the inoculation procedure easier. We examined
whether differences would be obtained in the antibody response according to the inoculation sites; the subcutaneous inoculation into the
back of the neck for OILVAX use, and the thigh, lower thigh, breast and shoulder muscle for the possible application of the inoculation
outdoors. The clear order was not found in the NDV-HI titer, IBV-SN titer against Nerima or TM-86 among the inoculation sites used
during the examination period. The IC serotypes A and C HI titers did vary among the inoculation sites; the subcutaneous inoculation
produced the highest antibody titer, and high antibody titers were observed in the order of lower thigh muscle $\geqq$ thigh muscle $>$ breast
muscle $\geqq$ shoulder muscle inoculation. - KEY wORDs: antibody response, chicken, inoculation site, oil-adjuvanted vaccine. J. Vet. Med. Sci. 60(7): 831-835, 1998

Newcastle disease (ND), infectious bronchitis (IB), and infectious coryza (IC) are major infectious diseases of chickens. Various live and inactivated vaccines have been used for the prevention of these diseases. For the inactivated vaccines, oil-adjuvanted vaccines have recently been employed instead of aluminum gel vaccines. A single inoculation of an oil-adjuvanted vaccine has been shown to induce a high level and long period of circulating antibodies without reinoculations [2, 6, 12, 13]. A disadvantage of this method was that several inoculations were necessary because oil-adjuvanted mixture vaccine was hardly marketed.

Windowless poultry housing is widely used in the poultry industry. In this breeding environment, it is difficult to administer reinoculations because of bad working position. Aluminum gel vaccines and oil-adjuvanted vaccines have been used together in an attempt to overcome this problem, but neither marketed vaccine has been completely overcome these problems. Therefore, the development of inactivated vaccines for various kinds of illness with which an oiladjuvant can be used is desirable.

Inactivated oil-adjuvanted vaccines for $\mathrm{ND}, \mathrm{IB}$, and IC serotypes $\mathrm{A}$ and $\mathrm{C}$ (OILVAX NB $\mathrm{N}_{2} \mathrm{AC}$ ) have been marketed from 1993. It has been anticipated that these vaccines will overcome the problems mentioned above. In the outdoors, various inoculation sites have been used in chickens because to make the inoculation procedure easier. We examined whether differences would be obtained in the antibody response according to the inoculation sites; the subcutaneous inoculation into the back of the neck for OILVAX use, and the thigh, lower thigh, breast and shoulder muscle for the possible application of the inoculation outdoors.

\section{MATERIALS AND METHODS}

Chickens: Breed A chickens (white, $\mathrm{n}=140$ ) and breed B chickens (brown, $n=140$ ) were used. Each chicken was primed with live IB vaccine at 0 day of age, live ND vaccine at 7 and 14 days of age, and live ND/IB combined vaccine at 28 days of age. Breed $\mathrm{A}$ was housed at 83 days of age in an individual battery cage, and breed $\mathrm{B}$ was at 82 days of age. Breed $\mathrm{A}$ was inoculated with OILVAX NB${ }_{2} \mathrm{AC}$ at 90 days of age, and breed $B$ was inoculated similarly at 89 days of age.

Organisms: The isolates of Newcastle disease virus (NDV) strain Ishii and Haemophilus paragallinarum (H.pg.) serotype A strain No.221 were provided by the National Veterinary Assay Laboratory, Tokyo, Japan. Infectious bronchitis virus (IBV) strain Nerima was provided by the National Institute of Animal Health, Ibaraki, Japan. Strain TM-86 of IBV and strain 53-47 of H.pg. serotype C were isolated from infected chickens in the Chemo-SeroTherapeutic Research Institute, Kumamoto, Japan. Strain Nerima and TM-86 of IBV, both of which belong to serotype $\mathrm{M}$, were shown to be antigenically different by a crossneutralization test. These strains were used for the preparation of the vaccines.

Preparation of vaccines (OILVAX NB ${ }_{2} A C$ ): NDV and IBV were inoculated 10- and 11-day-old embrionated chicken eggs, respectively. After incubation for 2 to 4 days at $37^{\circ} \mathrm{C}$, the allantoic fluids were harvested. H.pg. was cultured in chicken meat infusion broth medium supplemented with nicotinamide adenine dinucleotide and chicken serum at $37^{\circ} \mathrm{C}$. These cultures were inactivated by formalin and adjusted to appropriate concentrations. The oil adjuvant 
was added and the mixture was emulsified.

Immunization: First, $0.5 \mathrm{ml}$ of OILVAX $\mathrm{NB}_{2} \mathrm{AC}$ (Lot. 10) was inoculated either subcutaneously into the back of the neck or intramuscularly in the lower thigh, thigh, breast, or shoulder in 25 chickens each. In the OILVAX noninoculation group, 15 chickens of each breed were primed as described above.

Serology: Blood samples were collected from all birds before inoculation and at 1, 2, 3, 4, 6, 8, 10, 12 and 14 months post-inoculation (PI) and inactivated by heating at $56^{\circ} \mathrm{C}$ for $30 \mathrm{~min}$. Serum samples were maintained frozen at $-20^{\circ} \mathrm{C}$ until the antibody titers were measured. The antibody titers against the NDV $[1,11]$ and H.pg. serotypes $\mathrm{A}$ and $\mathrm{C}$ in sera individually were assayed by the HI test. H.pg. were assayed by HI test kits according to the instruction manuals from Nihon Pharmacy (Tochigi, Japan). IBV (Nerima and TM-86 strain) antibodies individually were determined with the serum-neutralization (SN) test using CK cell cultures [10].

Statistical analysis: The difference in various inoculation site groups were analyzed using the Mann-Whitney U-test. The results of the test were summarized with $P$ values. The $P$ values less than 0.05 were regarded as statistically significant.

\section{RESULTS}

Serial NDV-HI titer alterations: Table 1 shows the changes in the geometric mean (GM) of NDV-HI titers. In the OILVAX inoculation groups, the NDV-HI titer increased to a peak 1 month PI, and fell by degrees thereafter. In breed A, no significant difference was observed among the various inoculation site groups at 6 and 14 months PI. The subcutaneous, lower thigh and thigh muscle inoculation sites showed significantly higher titers than the breast and shoulder muscle site groups at 1, 2, 4 and 8 months PI. In breed B, no significant difference was observed among the various groups at $4,6,8,12$ and 14 months PI. In the two non-inoculation groups, the NDV-HI titers were 14 and 47 before the inoculation, gradually decreased thereafter, and were negative at 3 months PI. Wild type strain of NDV didn't infiltrate during examination period.

Serial changes in the IBV-SN titer against Nerima strain: Table 2 shows the changes in the IBV-SN titer against Nerima. In the OILVAX inoculation groups, the IBV-SN titers against Nerima increased to a peak 2 months PI, and gradually decreased thereafter. An increase in the antibody titer was recognized again from 8 months PI. In breed A, the subcutaneous, lower thigh and thigh muscle inoculation sites showed significantly higher titers than the breast and shoulder muscle sites at 2 and 4 months PI. At 1, 3 and 6 months PI, similar tendency was recognized. In breed B, the subcutaneous site showed significantly lower titer than the other site groups at 1 month PI. No significant difference was observed among the various groups at 2, 3, 4 and 6 months PI. In the non-inoculation groups, the IBV$\mathrm{SN}$ titers against Nerima were 10 and 11 before inoculation, fell by degrees afterward, and rose suddenly at 8 months PI. Thus, it was thought that the nearly wild-type strain of IBV Nerima induced an infection in this time. However, no clinical signs of IB were observed during the examination period.

Serial changes in the IBV-SN titer against TM-86 strain: Table 3 shows the changes in the IBV-SN titer against TM86. In the OILVAX inoculation groups, the IBV-SN titers against TM-86 increased to a peak 1 or 2 months PI, and declined thereafter; an increase in the antibody titer was seen again from 6 months PI. In both breeds, no significant difference was shown among the various inoculation site groups at 1, 2, 3 and 4 months PI. In the non-inoculation groups, the IBV-SN titers against TM-86 were $<2$ and 23 before inoculation, fell by degrees afterward in breed B, and rose suddenly at 6 months PI in both breeds. It was thus thought that the nearly wild-type strain of IBV TM-86 induced an infection in this time.

Serial changes in the IC serotype A HI titer: Table 4

Table 1. NDV-HI titers ${ }^{\text {a) }}$ before and after OILVAX inoculation at various sites in two breeds of chicken

\begin{tabular}{|c|c|c|c|c|c|c|c|c|c|c|c|}
\hline \multirow[t]{2}{*}{ Breed } & \multirow[t]{2}{*}{ Inoculations site } & \multicolumn{10}{|c|}{ Months after OILVAX inoculation } \\
\hline & & PRE & 1 & 2 & 3 & 4 & 6 & 8 & 10 & 12 & 14 \\
\hline \multirow{6}{*}{ A } & Shoulder muscle & $33.9^{\mathrm{a}}$ & $1391.0^{c}$ & $676.5^{\mathrm{b}}$ & $588.9^{\mathrm{b}}$ & $586.9^{\mathrm{b}}$ & $422.2^{\mathrm{a}}$ & $357.5^{\mathrm{b}}$ & $377.9^{\mathrm{ab}}$ & $372.0^{\mathrm{b}}$ & $391.7^{\mathrm{a}}$ \\
\hline & Breast muscle & $34.6^{\mathrm{a}}$ & $1736.5^{\mathrm{bc}}$ & $931.6^{\mathrm{b}}$ & $718.4^{\mathrm{ab}}$ & $798.9^{\mathrm{b}}$ & $446.3^{\mathrm{a}}$ & $361.0^{\mathrm{b}}$ & $367.6^{\mathrm{ab}}$ & $329.0^{\mathrm{b}}$ & $471.8^{\mathrm{a}}$ \\
\hline & Thigh muscle & $38.9^{\mathrm{a}}$ & $2050.7^{\mathrm{ab}}$ & $1708.6^{\mathrm{a}}$ & $970.1^{\mathrm{ab}}$ & $905.1^{\mathrm{ab}}$ & $588.9^{\mathrm{a}}$ & $439.7^{\mathrm{ab}}$ & $271.0^{\mathrm{b}}$ & $391.7^{\mathrm{b}}$ & $584.7^{\mathrm{a}}$ \\
\hline & Lower thigh muscle & $38.9^{\mathrm{a}}$ & $2782.0^{\mathrm{a}}$ & $1597.9^{\mathrm{a}}$ & $1177.8^{\mathrm{a}}$ & $975.9^{\mathrm{ab}}$ & $640.0^{\mathrm{a}}$ & $407.2^{\mathrm{ab}}$ & $415.0^{\mathrm{a}}$ & $465.8^{\mathrm{ab}}$ & $605.5^{\mathrm{a}}$ \\
\hline & Subcutaneous & $43.8^{\mathrm{a}}$ & $2632.0^{\mathrm{a}}$ & $1597.9^{\mathrm{a}}$ & $997.3^{\mathrm{ab}}$ & $1280.0^{\mathrm{a}}$ & $658.0^{\mathrm{a}}$ & $534.1^{\mathrm{a}}$ & $452.5^{\mathrm{a}}$ & $640.0^{\mathrm{a}}$ & $621.8^{\mathrm{a}}$ \\
\hline & Non-shot & $13.8^{\mathrm{b}}$ & $10.5^{\mathrm{d}}$ & $10.0^{c}$ & $<5^{c}$ & $<5^{\mathrm{c}}$ & $<5^{\mathrm{b}}$ & $<5^{\mathrm{c}}$ & $<5^{c}$ & $<5^{\mathrm{c}}$ & $<5^{\mathrm{b}}$ \\
\hline \multirow{6}{*}{ B } & Shoulder muscle & $71.3^{\mathrm{a}}$ & $2940.7^{b}$ & $2108.4^{\mathrm{ab}}$ & $1083.8^{\mathrm{b}}$ & $975.9^{\mathrm{a}}$ & $553.9^{\mathrm{a}}$ & $658.0^{\mathrm{a}}$ & $541.9^{\mathrm{b}}$ & $621.0^{\mathrm{a}}$ & $572.8^{\mathrm{a}}$ \\
\hline & Breast muscle & $71.3^{\mathrm{a}}$ & $4144.1^{\mathrm{ab}}$ & $1660.0^{c}$ & $1169.4^{\mathrm{b}}$ & $1145.6^{\mathrm{a}}$ & $622.5^{\mathrm{a}}$ & $586.9^{\mathrm{a}}$ & $452.5^{\mathrm{b}}$ & $744.1^{\mathrm{a}}$ & $640.0^{\mathrm{a}}$ \\
\hline & Thigh muscle & $82.2^{\mathrm{a}}$ & $4582.5^{\mathrm{a}}$ & $2560.0^{\mathrm{ab}}$ & $1940.1^{\mathrm{a}}$ & $1054.2^{\mathrm{a}}$ & $715.1^{\mathrm{a}}$ & $658.0^{\mathrm{a}}$ & $806.3^{\mathrm{a}}$ & $735.2^{\mathrm{a}}$ & $605.5^{\mathrm{a}}$ \\
\hline & Lower thigh muscle & $58.2^{\mathrm{a}}$ & $4101.5^{\mathrm{ab}}$ & $2782.0^{\mathrm{a}}$ & $1887.1^{\mathrm{a}}$ & $1177.8^{\mathrm{a}}$ & $695.5^{\mathrm{a}}$ & $695.5^{\mathrm{a}}$ & $422.2^{\mathrm{b}}$ & $695.5^{\mathrm{a}}$ & $557.2^{\mathrm{a}}$ \\
\hline & Subcutaneous & $51.3^{\mathrm{a}}$ & $5120.0^{\mathrm{a}}$ & $1835.5^{\mathrm{bc}}$ & $1177.8^{\mathrm{b}}$ & $997.3^{\mathrm{a}}$ & $715.1^{\mathrm{a}}$ & $513.3^{\mathrm{a}}$ & $419.7^{b}$ & $640.0^{\mathrm{a}}$ & $502.9^{\mathrm{a}}$ \\
\hline & Non-shot & $46.9^{\mathrm{a}}$ & $22.3^{\mathrm{c}}$ & $9.5^{\mathrm{d}}$ & $<5^{\mathrm{c}}$ & $<5^{\mathrm{b}}$ & $<5^{\mathrm{b}}$ & $<5^{\mathrm{b}}$ & $<5^{\mathrm{c}}$ & $<5^{\mathrm{b}}$ & $<5^{\mathrm{b}}$ \\
\hline
\end{tabular}

a) Geometric mean titer. Titers within a column followed by different lower-case superscripts are significantly different $(\mathrm{P}<0.05) .<5$; All samples were negative. 
Table 2. IBV-SN titers against strain of Nerima ${ }^{\text {a) }}$ before and after OILVAX inoculation at various sites in two breeds of chicken

\begin{tabular}{|c|c|c|c|c|c|c|c|c|c|c|c|}
\hline \multirow[t]{2}{*}{ Breed } & \multirow[t]{2}{*}{ Inoculations site } & \multicolumn{10}{|c|}{ Months after OILVAX inoculation } \\
\hline & & PRE & 1 & 2 & 3 & 4 & 6 & 8 & 10 & 12 & 14 \\
\hline \multirow{6}{*}{ A } & Shoulder muscle & $10.0^{\mathrm{ab}}$ & $67.6^{\mathrm{c}}$ & $86.8^{\mathrm{c}}$ & $43.4^{\mathrm{b}}$ & $39.2^{\mathrm{b}}$ & $22.9^{\mathrm{b}}$ & $430.5^{b}$ & $632.2^{\mathrm{a}}$ & $780.7^{\mathrm{a}}$ & $829.2^{\mathrm{a}}$ \\
\hline & Breast muscle & $9.4^{\mathrm{ab}}$ & $79.9^{c}$ & $209.1^{\mathrm{b}}$ & $70.1^{\mathrm{ab}}$ & $55.7^{\mathrm{b}}$ & $46.6^{\mathrm{ab}}$ & $467.7^{b}$ & $812.7^{\mathrm{a}}$ & $1060.1^{\mathrm{a}}$ & $989.1^{\mathrm{a}}$ \\
\hline & Thigh muscle & $7.4^{\mathrm{b}}$ & $398.5^{\mathrm{a}}$ & $473.9^{\mathrm{a}}$ & $112.2^{\mathrm{a}}$ & $110.8^{\mathrm{a}}$ & $70.3^{\mathrm{a}}$ & $1090.6^{\mathrm{a}}$ & $955.4^{\mathrm{a}}$ & $912.3^{\mathrm{a}}$ & $1024.0^{\mathrm{a}}$ \\
\hline & Lower thigh muscle & $8.5^{\mathrm{b}}$ & $183.5^{\mathrm{b}}$ & $367.5^{\mathrm{ab}}$ & $45.3^{\mathrm{b}}$ & $60.3^{\mathrm{ab}}$ & $52.0^{\mathrm{ab}}$ & $427.3^{\mathrm{b}}$ & $664.0^{\mathrm{a}}$ & $829.2^{\mathrm{a}}$ & $922.9^{\mathrm{a}}$ \\
\hline & Subcutaneous & $12.5^{\mathrm{a}}$ & $367.1^{\mathrm{ab}}$ & $385.6^{\mathrm{a}}$ & $90.5^{\mathrm{a}}$ & $65.9^{\mathrm{ab}}$ & $50.8^{\mathrm{ab}}$ & $339.9^{\mathrm{b}}$ & $703.5^{\mathrm{a}}$ & $891.4^{\mathrm{a}}$ & $724.1^{\mathrm{a}}$ \\
\hline & Non-shot & $10.4^{\mathrm{ab}}$ & $27.6^{\mathrm{d}}$ & $18.8^{\mathrm{d}}$ & $11.0^{\mathrm{c}}$ & $8.9^{c}$ & $5.2^{\mathrm{c}}$ & $199.0^{\mathrm{b}}$ & $600.8^{\mathrm{a}}$ & $877.8^{\mathrm{a}}$ & $1024.0^{\mathrm{a}}$ \\
\hline \multirow{6}{*}{ B } & Shoulder muscle & $10.6^{\mathrm{a}}$ & $105.4^{\mathrm{ab}}$ & $183.5^{\mathrm{a}}$ & $71.5^{\mathrm{a}}$ & $55.7^{\mathrm{a}}$ & $35.5^{\mathrm{a}}$ & $77.7^{\mathrm{c}}$ & $94.7^{c}$ & $116.5^{\mathrm{b}}$ & $175.4^{\mathrm{cd}}$ \\
\hline & Breast muscle & $10.6^{\mathrm{a}}$ & $101.6^{\mathrm{ab}}$ & $234.8^{\mathrm{a}}$ & $73.5^{\mathrm{a}}$ & $41.1^{\mathrm{a}}$ & $45.9^{\mathrm{a}}$ & $156.7^{\mathrm{bc}}$ & $213.7^{\mathrm{b}}$ & $282.6^{\mathrm{ab}}$ & $319.6^{\mathrm{bc}}$ \\
\hline & Thigh muscle & $11.8^{\mathrm{a}}$ & $76.7^{\mathrm{ab}}$ & $124.4^{\mathrm{a}}$ & $70.7^{\mathrm{a}}$ & $45.9^{\mathrm{a}}$ & $27.9^{\mathrm{a}}$ & $421.7^{\mathrm{a}}$ & $367.1^{\mathrm{ab}}$ & $286.0^{\mathrm{ab}}$ & $433.5^{\mathrm{ab}}$ \\
\hline & Lower thigh muscle & $11.8^{\mathrm{a}}$ & $128.0^{\mathrm{a}}$ & $222.9^{\mathrm{a}}$ & $84.4^{\mathrm{a}}$ & $42.2^{\mathrm{a}}$ & $42.0^{\mathrm{a}}$ & $256.0^{\mathrm{ab}}$ & $397.9^{\mathrm{ab}}$ & $467.7^{\mathrm{a}}$ & $380.4^{\mathrm{b}}$ \\
\hline & Subcutaneous & $10.3^{\mathrm{a}}$ & $45.3^{\mathrm{bc}}$ & $175.9^{\mathrm{a}}$ & $78.0^{\mathrm{a}}$ & $52.3^{\mathrm{a}}$ & $30.2^{\mathrm{a}}$ & $124.2^{\mathrm{bc}}$ & $164.3^{\mathrm{bc}}$ & $175.4^{\mathrm{b}}$ & $154.6^{\mathrm{d}}$ \\
\hline & Non-shot & $11.0^{\mathrm{a}}$ & $19.8^{\mathrm{c}}$ & $16.9^{\mathrm{b}}$ & $7.2^{\mathrm{b}}$ & $7.6^{\mathrm{b}}$ & $3.5^{\mathrm{b}}$ & $203.2^{\mathrm{ab}}$ & $436.3^{\mathrm{a}}$ & $658.8^{\mathrm{a}}$ & $827.3^{\mathrm{a}}$ \\
\hline
\end{tabular}

a) Geometric mean titer. Titers within a column followed by different lower-case superscripts are significantly different $(\mathrm{P}<0.05)$.

Table 3. IBV-SN titers against strain of TM-86a) before and after OILVAX inoculation at various sites in two breeds of chicken

\begin{tabular}{|c|c|c|c|c|c|c|c|c|c|c|c|}
\hline \multirow[t]{2}{*}{ Breed } & \multirow[t]{2}{*}{ Inoculations site } & \multicolumn{10}{|c|}{ Months after OILVAX inoculation } \\
\hline & & PRE & 1 & 2 & 3 & 4 & 6 & 8 & 10 & 12 & 14 \\
\hline \multirow{6}{*}{ A } & Shoulder muscle & $7.6^{\mathrm{c}}$ & $178.3^{\mathrm{a}}$ & $149.8^{\mathrm{a}}$ & $99.7^{\mathrm{a}}$ & $85.4^{\mathrm{a}}$ & $58.5^{\mathrm{a}}$ & $135.3^{\mathrm{ab}}$ & $542.4^{\mathrm{a}}$ & $1168.5^{\mathrm{a}}$ & $1264.5^{\mathrm{a}}$ \\
\hline & Breast muscle & $10.0^{\mathrm{bc}}$ & $164.3^{\mathrm{a}}$ & $145.2^{\mathrm{a}}$ & $106.8^{\mathrm{a}}$ & $73.5^{\mathrm{a}}$ & $60.4^{\mathrm{a}}$ & $220.2^{\mathrm{ab}}$ & $671.5^{\mathrm{a}}$ & $1351.2^{\mathrm{a}}$ & $1579.2^{\mathrm{a}}$ \\
\hline & Thigh muscle & $13.2^{\mathrm{b}}$ & $203.1^{\mathrm{a}}$ & $162.8^{\mathrm{a}}$ & $148.8^{\mathrm{a}}$ & $111.4^{\mathrm{a}}$ & $64.0^{\mathrm{a}}$ & $264.6^{\mathrm{a}}$ & $829.2^{\mathrm{a}}$ & $1749.5^{\mathrm{a}}$ & $1815.2^{\mathrm{a}}$ \\
\hline & Lower thigh muscle & $8.7^{\mathrm{bc}}$ & $149.8^{\mathrm{a}}$ & $149.8^{\mathrm{a}}$ & $139.1^{\mathrm{a}}$ & $97.0^{\mathrm{a}}$ & $58.9^{\mathrm{a}}$ & $136.0^{\mathrm{ab}}$ & $724.1^{\mathrm{a}}$ & $1974.6^{\mathrm{a}}$ & $1782.9^{\mathrm{a}}$ \\
\hline & Subcutaneous & $11.8^{\mathrm{bc}}$ & $205.7^{\mathrm{a}}$ & $211.9^{\mathrm{a}}$ & $167.9^{\mathrm{a}}$ & $97.6^{\mathrm{a}}$ & $62.2^{\mathrm{a}}$ & $102.7^{\mathrm{b}}$ & $471.1^{\mathrm{a}}$ & $985.3^{\mathrm{a}}$ & $1060.1^{\mathrm{a}}$ \\
\hline & Non-shot & $23.2^{\mathrm{a}}$ & $12.5^{\mathrm{b}}$ & $7.2^{\mathrm{b}}$ & $3.4^{\mathrm{b}}$ & $6.5^{\mathrm{b}}$ & $72.6^{\mathrm{a}}$ & $136.3^{\mathrm{ab}}$ & $512.0^{\mathrm{a}}$ & $1024.0^{\mathrm{a}}$ & $1328.0^{\mathrm{a}}$ \\
\hline \multirow{6}{*}{ B } & Shoulder muscle & $<2^{\mathrm{a}}$ & $32.9^{\mathrm{a}}$ & $24.9^{\mathrm{a}}$ & $20.0^{\mathrm{a}}$ & $15.1^{\mathrm{a}}$ & $12.1^{\mathrm{b}}$ & $41.1^{\mathrm{c}}$ & $230.7^{\mathrm{a}}$ & $584.3^{\mathrm{ab}}$ & $780.7^{\mathrm{b}}$ \\
\hline & Breast muscle & $<2^{\mathrm{a}}$ & $24.9^{\mathrm{a}}$ & $22.3^{\mathrm{a}}$ & $16.9^{\mathrm{a}}$ & $14.7^{\mathrm{a}}$ & $11.5^{\mathrm{b}}$ & $139.6^{\mathrm{ab}}$ & $498.0^{\mathrm{a}}$ & $1024.0^{\mathrm{a}}$ & $1351.2^{\mathrm{ab}}$ \\
\hline & Thigh muscle & $<2^{\mathrm{a}}$ & $33.8^{\mathrm{a}}$ & $24.9^{\mathrm{a}}$ & $15.1^{\mathrm{a}}$ & $15.1^{\mathrm{a}}$ & $14.3^{\mathrm{b}}$ & $319.6^{\mathrm{a}}$ & $604.7^{\mathrm{a}}$ & $1217.7^{\mathrm{a}}$ & $1470.1^{\mathrm{a}}$ \\
\hline & Lower thigh muscle & $<2^{\mathrm{a}}$ & $29.4^{\mathrm{a}}$ & $31.1^{\mathrm{a}}$ & $21.7^{\mathrm{a}}$ & $20.0^{\mathrm{a}}$ & $20.0^{\mathrm{b}}$ & $434.1^{\mathrm{a}}$ & $512.0^{\mathrm{a}}$ & $735.1^{\mathrm{ab}}$ & $891.4^{\mathrm{b}}$ \\
\hline & Subcutaneous & $<2^{\mathrm{a}}$ & $45.9^{\mathrm{a}}$ & $34.8^{\mathrm{a}}$ & $24.9^{\mathrm{a}}$ & $21.1^{\mathrm{a}}$ & $20.0^{\mathrm{b}}$ & $79.0^{\mathrm{bc}}$ & $211.9^{\mathrm{a}}$ & $322.5^{\mathrm{b}}$ & $591.5^{\mathrm{b}}$ \\
\hline & Non-shot & $<2^{\mathrm{a}}$ & $2.2^{\mathrm{b}}$ & $2.2^{\mathrm{b}}$ & $<2^{\mathrm{b}}$ & $2.8^{\mathrm{b}}$ & $77.0^{\mathrm{a}}$ & $256.0^{\mathrm{a}}$ & $707.5^{\mathrm{a}}$ & $1237.1^{\mathrm{a}}$ & $1521.7^{\mathrm{a}}$ \\
\hline
\end{tabular}

a) Geometric mean titer. Titers within a column followed by different lower-case superscripts are significantly different $(\mathrm{P}<0.05)$. $<2$; All samples were negative.

shows the changes in the IC serotype A HI titers. In the OILVAX inoculation groups, the IC serotype A HI titers were negative before inoculation, increased to a peak 2 months PI, and declined thereafter. The antibody titer varied greatly by inoculation sites in both breeds. In breed $\mathrm{A}$, the subcutaneous, lower thigh and thigh muscle inoculation sites showed significantly higher titers than the breast and shoulder muscle sites at 3, 4, 6, 10 and 12 months PI. In breed $\mathrm{B}$, high antibody titers were seen in the following (descending) order: subcutaneously into the back of the neck $\geqq$ intramuscularly in lower thigh, thigh $>$ intramuscularly in breast, shoulder in all examinations period. In the noninoculation groups, the IC serotype A HI titers were negative during the entire examination period.

Serial changes in the IC serotype $C H I$ titer: Table 5 shows the changes in the IC serotype $\mathrm{C} \mathrm{HI}$ titers. In the
OILVAX inoculation groups, the IC serotype C HI titers were negative before inoculation, increased to a peak 2 months PI, and declined thereafter. The antibody titer varied greatly by inoculation sites in both breeds. In breed A, the group inoculated subcutaneously into the back of the neck had significantly higher titers than the other groups at 1,2 , 4, 6 and 8 months PI. The subcutaneous and lower thigh muscle groups had significantly higher titers than the other groups at 10, 12 and 14 months PI. In breed B, the subcutaneous and lower thigh muscle inoculation site groups had significantly higher titers than the other groups at 1, 4, 6 and 12 months PI. The subcutaneous, lower thigh and thigh muscle groups showed higher titers than the breast and shoulder muscle groups at 2, 3, 10 and 14 months PI. In the non-inoculation groups, the IC serotype $\mathrm{C}$ HI titers were negative during the examination period. 
Table 4. IC serotype A HI titers ${ }^{\text {a) }}$ before and after OILVAX inoculation at various sites in two breeds of chicken

\begin{tabular}{|c|c|c|c|c|c|c|c|c|c|c|c|}
\hline \multirow[t]{2}{*}{ Breed } & \multirow[t]{2}{*}{ Inoculations site } & \multicolumn{10}{|c|}{ Months after OILVAX inoculation } \\
\hline & & PRE & 1 & 2 & 3 & 4 & 6 & 8 & 10 & 12 & 14 \\
\hline \multirow{6}{*}{ A } & Shoulder muscle & $<5^{\mathrm{a}}$ & $12.8^{\mathrm{b}}$ & $27.1^{\mathrm{c}}$ & $29.1^{\mathrm{b}}$ & $16.0^{\mathrm{c}}$ & $21.9^{\mathrm{c}}$ & $12.8^{\mathrm{a}}$ & $10.0^{\mathrm{bc}}$ & $5.3^{\mathrm{bc}}$ & $4.6^{\mathrm{c}}$ \\
\hline & Breast muscle & $<5^{\mathrm{a}}$ & $8.7^{\mathrm{c}}$ & $19.4^{\mathrm{c}}$ & $25.5^{\mathrm{b}}$ & $23.6^{\mathrm{bc}}$ & $23.3^{\mathrm{bc}}$ & $5.8^{\mathrm{b}}$ & $8.6^{\mathrm{c}}$ & $4.5^{\mathrm{c}}$ & $5.0^{\mathrm{bc}}$ \\
\hline & Thigh muscle & $<5^{\mathrm{a}}$ & $10.6^{\mathrm{bc}}$ & $54.1^{\mathrm{b}}$ & $38.9^{\mathrm{ab}}$ & $37.8^{\mathrm{ab}}$ & $41.2^{\mathrm{a}}$ & $7.5^{\mathrm{b}}$ & $24.2^{\mathrm{a}}$ & $7.5^{\mathrm{ab}}$ & $6.1^{\mathrm{bc}}$ \\
\hline & Lower thigh muscle & $<5^{\mathrm{a}}$ & $13.2^{\mathrm{b}}$ & $59.4^{\mathrm{b}}$ & $36.4^{\mathrm{ab}}$ & $29.6^{\mathrm{ab}}$ & $47.6^{\mathrm{a}}$ & $7.6^{\mathrm{b}}$ & $16.8^{\mathrm{ab}}$ & $7.4^{\mathrm{ab}}$ & $7.0^{\mathrm{b}}$ \\
\hline & Subcutaneous & $<5^{\mathrm{a}}$ & $49.9^{\mathrm{a}}$ & $178.8^{\mathrm{a}}$ & $65.4^{\mathrm{a}}$ & $54.8^{\mathrm{a}}$ & $37.8^{\mathrm{ab}}$ & $20.6^{\mathrm{a}}$ & $25.8^{\mathrm{a}}$ & $11.0^{\mathrm{a}}$ & $20.0^{\mathrm{a}}$ \\
\hline & Non-shot & $<5^{\mathrm{a}}$ & $<5^{\mathrm{d}}$ & $<5^{\mathrm{d}}$ & $<5^{c}$ & $<5^{\mathrm{d}}$ & $<5^{\mathrm{d}}$ & $<5^{\mathrm{c}}$ & $<5^{\mathrm{d}}$ & $<5^{\mathrm{d}}$ & $<5^{\mathrm{d}}$ \\
\hline \multirow{6}{*}{ B } & Shoulder muscle & $<5^{\mathrm{a}}$ & $41.1^{\mathrm{c}}$ & $48.6^{c}$ & $25.0^{\mathrm{c}}$ & $16.9^{\mathrm{c}}$ & $10.7^{\mathrm{b}}$ & $6.2^{\mathrm{c}}$ & $6.5^{\mathrm{c}}$ & $4.2^{\mathrm{c}}$ & $4.5^{\mathrm{c}}$ \\
\hline & Breast muscle & $<5^{\mathrm{a}}$ & $55.0^{\mathrm{c}}$ & $55.8^{\mathrm{c}}$ & $29.5^{\mathrm{c}}$ & $17.4^{\mathrm{c}}$ & $11.3^{\mathrm{b}}$ & $5.9^{\mathrm{c}}$ & $6.0^{\mathrm{c}}$ & $4.0^{\mathrm{c}}$ & $3.8^{\mathrm{c}}$ \\
\hline & Thigh muscle & $<5^{\mathrm{a}}$ & $91.9^{\mathrm{bc}}$ & $242.5^{\mathrm{b}}$ & $80.0^{\mathrm{ab}}$ & $34.8^{\mathrm{b}}$ & $24.5^{\mathrm{a}}$ & $13.2^{\mathrm{b}}$ & $11.2^{\mathrm{b}}$ & $8.0^{\mathrm{b}}$ & $8.2^{\mathrm{b}}$ \\
\hline & Lower thigh muscle & $<5^{\mathrm{a}}$ & $143.2^{\mathrm{b}}$ & $286.4^{\mathrm{ab}}$ & $57.4^{\mathrm{b}}$ & $38.9^{\mathrm{b}}$ & $20.6^{\mathrm{a}}$ & $12.5^{\mathrm{b}}$ & $10.9^{\mathrm{b}}$ & $8.9^{\mathrm{b}}$ & $10.3^{\mathrm{b}}$ \\
\hline & Subcutaneous & $<5^{\mathrm{a}}$ & $329.0^{\mathrm{a}}$ & $465.8^{\mathrm{a}}$ & $93.0^{\mathrm{a}}$ & $71.6^{\mathrm{a}}$ & $30.1^{\mathrm{a}}$ & $31.4^{\mathrm{a}}$ & $28.8^{\mathrm{a}}$ & $15.2^{\mathrm{a}}$ & $17.6^{\mathrm{a}}$ \\
\hline & Non-shot & $<5^{\mathrm{a}}$ & $<5^{\mathrm{d}}$ & $<5^{\mathrm{d}}$ & $<5^{\mathrm{d}}$ & $<5^{\mathrm{d}}$ & $<5^{\mathrm{c}}$ & $<5^{\mathrm{d}}$ & $<5^{\mathrm{d}}$ & $<5^{\mathrm{d}}$ & $<5^{\mathrm{d}}$ \\
\hline
\end{tabular}

a) Geometric mean titer. Titers within a column followed by different lower-case superscripts are significantly different $(\mathrm{P}<0.05)$. $<5$; All samples were negative.

Table 5. IC serotype C HI titers ${ }^{\text {a) }}$ before and after OILVAX inoculation at various sites in two breeds of chicken

\begin{tabular}{|c|c|c|c|c|c|c|c|c|c|c|c|}
\hline \multirow[t]{2}{*}{ Breed } & \multirow[t]{2}{*}{ Inoculations site } & \multicolumn{10}{|c|}{ Months after OILVAX inoculation } \\
\hline & & PRE & 1 & 2 & 3 & 4 & 6 & 8 & 10 & 12 & 14 \\
\hline \multirow{6}{*}{ A } & Shoulder muscle & $<5^{\mathrm{a}}$ & $6.6^{\mathrm{b}}$ & $10.0^{\mathrm{c}}$ & $12.2^{\mathrm{b}}$ & $9.7^{\mathrm{b}}$ & $5.9^{\mathrm{bc}}$ & $5.0^{\mathrm{b}}$ & $<5^{\mathrm{c}}$ & $<5^{\mathrm{c}}$ & $<5^{\mathrm{b}}$ \\
\hline & Breast muscle & $<5^{\mathrm{a}}$ & $5.6^{\mathrm{b}}$ & $11.2^{\mathrm{bc}}$ & $11.3^{\mathrm{b}}$ & $10.3^{\mathrm{b}}$ & $5.2^{\mathrm{c}}$ & $5.0^{\mathrm{b}}$ & $2.6^{\mathrm{bc}}$ & $2.6^{\mathrm{bc}}$ & $2.6^{\mathrm{b}}$ \\
\hline & Thigh muscle & $<5^{\mathrm{a}}$ & $5.9^{\mathrm{b}}$ & $12.6^{\mathrm{bc}}$ & $13.5^{\mathrm{ab}}$ & $11.6^{\mathrm{b}}$ & $5.7^{b c}$ & $5.0^{\mathrm{b}}$ & $2.6^{\mathrm{bc}}$ & $2.6^{\mathrm{bc}}$ & $2.7^{\mathrm{b}}$ \\
\hline & Lower thigh muscle & $<5^{\mathrm{a}}$ & $6.6^{\mathrm{b}}$ & $15.9^{\mathrm{b}}$ & $10.9^{\mathrm{b}}$ & $12.2^{\mathrm{b}}$ & $6.9^{\mathrm{b}}$ & $5.2^{\mathrm{b}}$ & $3.1^{\mathrm{ab}}$ & $2.9^{\mathrm{ab}}$ & $2.8^{\mathrm{ab}}$ \\
\hline & Subcutaneous & $<5^{\mathrm{a}}$ & $13.2^{\mathrm{a}}$ & $42.3^{\mathrm{a}}$ & $24.5^{\mathrm{a}}$ & $32.7^{\mathrm{a}}$ & $16.4^{\mathrm{a}}$ & $7.5^{\mathrm{a}}$ & $4.7^{\mathrm{a}}$ & $4.2^{\mathrm{a}}$ & $4.2^{\mathrm{a}}$ \\
\hline & Non-shot & $<5^{\mathrm{a}}$ & $<5^{\mathrm{c}}$ & $<5^{\mathrm{d}}$ & $<5^{\mathrm{c}}$ & $<5^{\mathrm{c}}$ & $<5^{\mathrm{d}}$ & $<5^{\mathrm{c}}$ & $<5^{\text {bc }}$ & $<5^{\text {bc }}$ & $<5^{\mathrm{b}}$ \\
\hline \multirow{6}{*}{ B } & Shoulder muscle & $<5^{\mathrm{a}}$ & $20.6^{\mathrm{c}}$ & $25.0^{\mathrm{b}}$ & $21.1^{\mathrm{b}}$ & $14.3^{\mathrm{c}}$ & $7.4^{\mathrm{cd}}$ & $5.6^{\mathrm{c}}$ & $3.0^{\mathrm{b}}$ & $3.0^{\mathrm{c}}$ & $2.6^{\mathrm{b}}$ \\
\hline & Breast muscle & $<5^{\mathrm{a}}$ & $23.1^{\mathrm{bc}}$ & $41.2^{\mathrm{b}}$ & $25.8^{\mathrm{b}}$ & $13.2^{\mathrm{c}}$ & $6.0^{\mathrm{d}}$ & $5.6^{\mathrm{c}}$ & $2.9^{\mathrm{b}}$ & $2.9^{\mathrm{c}}$ & $2.8^{\mathrm{b}}$ \\
\hline & Thigh muscle & $<5^{\mathrm{a}}$ & $43.5^{\mathrm{b}}$ & $94.5^{\mathrm{a}}$ & $80.0^{\mathrm{a}}$ & $25.7^{\mathrm{b}}$ & $9.7^{\mathrm{bc}}$ & $8.5^{\mathrm{ab}}$ & $5.3^{\mathrm{a}}$ & $4.5^{\mathrm{b}}$ & $4.6^{\mathrm{a}}$ \\
\hline & Lower thigh muscle & $<5^{\mathrm{a}}$ & $102.7^{\mathrm{a}}$ & $155.6^{\mathrm{a}}$ & $67.7^{\mathrm{a}}$ & $30.3^{\mathrm{ab}}$ & $12.6^{\mathrm{ab}}$ & $8.0^{\mathrm{b}}$ & $5.3^{\mathrm{a}}$ & $5.2^{\mathrm{ab}}$ & $4.6^{\mathrm{a}}$ \\
\hline & Subcutaneous & $<5^{\mathrm{a}}$ & $169.1^{\mathrm{a}}$ & $121.3^{\mathrm{a}}$ & $64.8^{\mathrm{a}}$ & $41.1^{\mathrm{a}}$ & $16.0^{\mathrm{a}}$ & $12.3^{\mathrm{a}}$ & $7.5^{\mathrm{a}}$ & $8.0^{\mathrm{a}}$ & $7.1^{\mathrm{a}}$ \\
\hline & Non-shot & $<5^{\mathrm{a}}$ & $<5^{\mathrm{d}}$ & $<5^{\mathrm{c}}$ & $<5^{\mathrm{c}}$ & $<5^{\mathrm{d}}$ & $<5^{\mathrm{e}}$ & $<5^{\mathrm{d}}$ & $<5^{\mathrm{b}}$ & $<5^{\mathrm{c}}$ & $<5^{\mathrm{b}}$ \\
\hline
\end{tabular}

a) Geometric mean titer. Titers within a column followed by different lower-case superscripts are significantly different $(\mathrm{P}<0.05)$. $<5$;All samples were negative.

\section{DISCUSSION}

In the present study, we examined whether differences would be obtained in the antibody response according to the inoculation sites; the subcutaneous inoculation into the back of the neck for OILVAX use, and the thigh, lower thigh, breast and shoulder muscle for the possible application of the inoculation outdoors. The clear order was not found in the NDV-HI titer, IBV-SN titer against Nerima or TM-86 among the inoculation sites used during the examination period. The IC serotypes A and C HI titers did vary among the inoculation sites; the subcutaneous inoculation produced the highest antibody titer, and high antibody titers were observed in the order of lower thigh muscle $\geqq$ thigh muscle $>$ breast muscle $\geqq$ shoulder muscle inoculation.

The oil-adjuvanted vaccine gives some damages to chicken. Each site has some advantages and disadvantages. The shoulder muscle inoculation is difficult work because this muscle is comparatively smaller. Droual et al. [3] reported that the breast muscle inoculation frequently leads to residual lesions. The lesions typically are yellow opaque cysts along the fascial planes separating the superficial and deep pectoral muscles. The leg muscle inoculation [4] is often associated with lameness, and remains some cysts until processing. Local vaccine reaction typically involved swelling due to a granulomatous cellulitis in the connective tissue above the tibiotarsal joint of the vaccinated leg. As for the muscle inoculation, there is a problem at a point of safety. On the contrary, the local reaction of subcutaneous inoculation is not severer than the that of muscle. Especially, because OILVAX $\mathrm{NB}_{2} \mathrm{AC}$ used in the present study gives low viscous (approximately $20 \mathrm{cp}$ at $25^{\circ} \mathrm{C}$ ) that is secure inoculation and easy handling, the stress to chickens is few. In inoculation work, the leg site is easy to pull out a leg from a cage and can be done by one person. About the other inoculation sites, because it is inoculated 
after having taken out every 1 from a cage, it is troublesome. As a conclusion, although inoculation work is complicated, the subcutaneous inoculation into the back of the neck for OILVAX use is better route in view of safety and antibody response in chickens.

Levy and Zakay-Rones [8] inoculated chickens with inactivated oil-adjuvanted vaccine for ND subcutaneously or into muscle, and compared these sites for antibody response. The adjuvant of this vaccine was the classic oil adjuvant FIA (Freund's incomplete adjuvant). FIA has the disadvantage of large local reaction. Levy and Zakay-Rones reported that there was no difference between the inoculation sites regarding the NDV-HI titer. Their results agree with our present findings.

Iritani et al. [5] and Kuri et al. [7] inoculated chickens with an inactivated combined vaccine for ND and IC serotypes $\mathrm{A}$ and $\mathrm{C}$ into the breast muscle and thigh muscle, and compared these sites for each antibody response. They reported that there was no difference between the inoculation sites in the NDV-HI titer, and that the thigh muscle inoculation showed higher IC serotypes A and C HI titers than the breast muscle inoculation. Their results are in agreement with ours.

Mutalib and Boyle [9] inoculated chickens with an inactivated combined vaccine for ND and IB subcutaneously and into the breast, thigh and wing muscle, and compared the sites for each enzyme-linked immunosorbent assay titers. They reported that the subcutaneous and wing muscle inoculation sites produced significantly high NDV titers, and that there was no difference among the inoculation sites for IBV titers. They concluded that the difference of NDV antibody titers was not important, because the difference in titer per inoculation site was dependent on the size of antibody titer before the inoculation of inactivated vaccine.

Iritani et al. [5] reported that the difference of immunity effect by inoculation sites was a characteristic phenomenon in IC vaccine. Because an antigen is a bacillus, as is the case for IC, the size of the H.pg. is larger than that of a virus. This difference in size influences the diffusion in the interior of the body of the antigen, and may thus account for the difference in the antibody response.

\section{REFERENCES}

1. Allan, W.H. and Gough, R.E. 1974. A standard haemagglutination inhibition test for Newcastle disease. Vet. Rec. 95: 120-123.

2. Blackall, P.J., Eaves, L.E., Rogers, D.G. and Firth, G. 1992. An evaluation of inactivated infectious coryza vaccines containing a double-emulsion adjuvant system. Avian Dis. 36: 632-636.

3. Droual, R., Bickford, A.A., Charlton, B.R. and Kuney, D.R. 1990. Investigation of problems associated with intramuscular breast injection of oil-adjuvanted killed vaccines in chickens. Avian Dis. 34: 473-478.

4. Droual, R., Bickford, A.A. and Cutler, Gregg J. 1993. Local reaction and serological response in commercial layer chickens injected intramuscularly in the leg with oil-adjuvanted mycoplasma gallisepticum bacterin. Avian Dis. 37: 1001-1008.

5. Iritani, Y., Kunihiro, Y., Yamaguchi, T., Tomii, T. and Hayashi, Y. 1984. Difference of immune efficacy of infectious coryza vaccine by different site of injection in chickens. J. Jpn. Soc. Poult. Dis. 20: 182-185 (in Japanese).

6. Jacobs, A.A.C., Cuenen, W. and Storm, P.K. 1992. Efficacy of a trivalent Haemophilus paragallinarum vaccine compared to bivalent vaccines. Vet. Microbiol. 32: 43-49.

7. Kuri, S., Amano, T., Takeuchi, Y. and Inoue, H. 1987. Immune efficacy with the infectious coryza vaccinations at different regions and period. J. Jpn. Soc. Poult. Dis. 23: 2125 (in Japanese).

8. Levy, R. and Zakay-Rones, Z. 1973. Immunization of chickens with an inactivated oil-adjuvant Newcastle disease virus vaccine. Avian Dis. 17: 598-604.

9. Mutalib, A. and Boyle, C. R. 1994. Influence of site of inoculation of inactivated vaccines on the immune response in chickens. Avian Dis. 38: 857-860.

10. Page, C.A. and Cunningham, C.H. 1962. The neutralization test for infectious bronchitis virus. Am. J. Vet. Res. 23: 10651071.

11. Sever, J.L. 1962. Application of a microtechnique to viral serological investigation. J. Immunol. 88: 320-329.

12. Yamanaka, M., Okabe, T., Nakai, M. and Goto, N. 1993. Local pathological reactions and immune response of chickens to ISA-70 and other adjuvants containing Newcastle disease virus antigen. Avian Dis. 37: 459-466.

13. Zhixun Xie and Henry D. Stone. 1990. Immune response to oil-emulsion vaccines with single or mixed antigens of Newcastle disease, avian influenza, and infectious bronchitis. Avian Dis. 34: 154-162. 